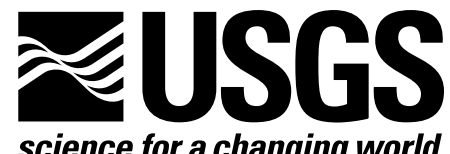

science for a changing world

\title{
A Preliminary Finite-Element Analysis of Wave Propagation in a Multistory Building
}

By W.Z. Savage and E. Safak

Open-File Report 01-0057

2001

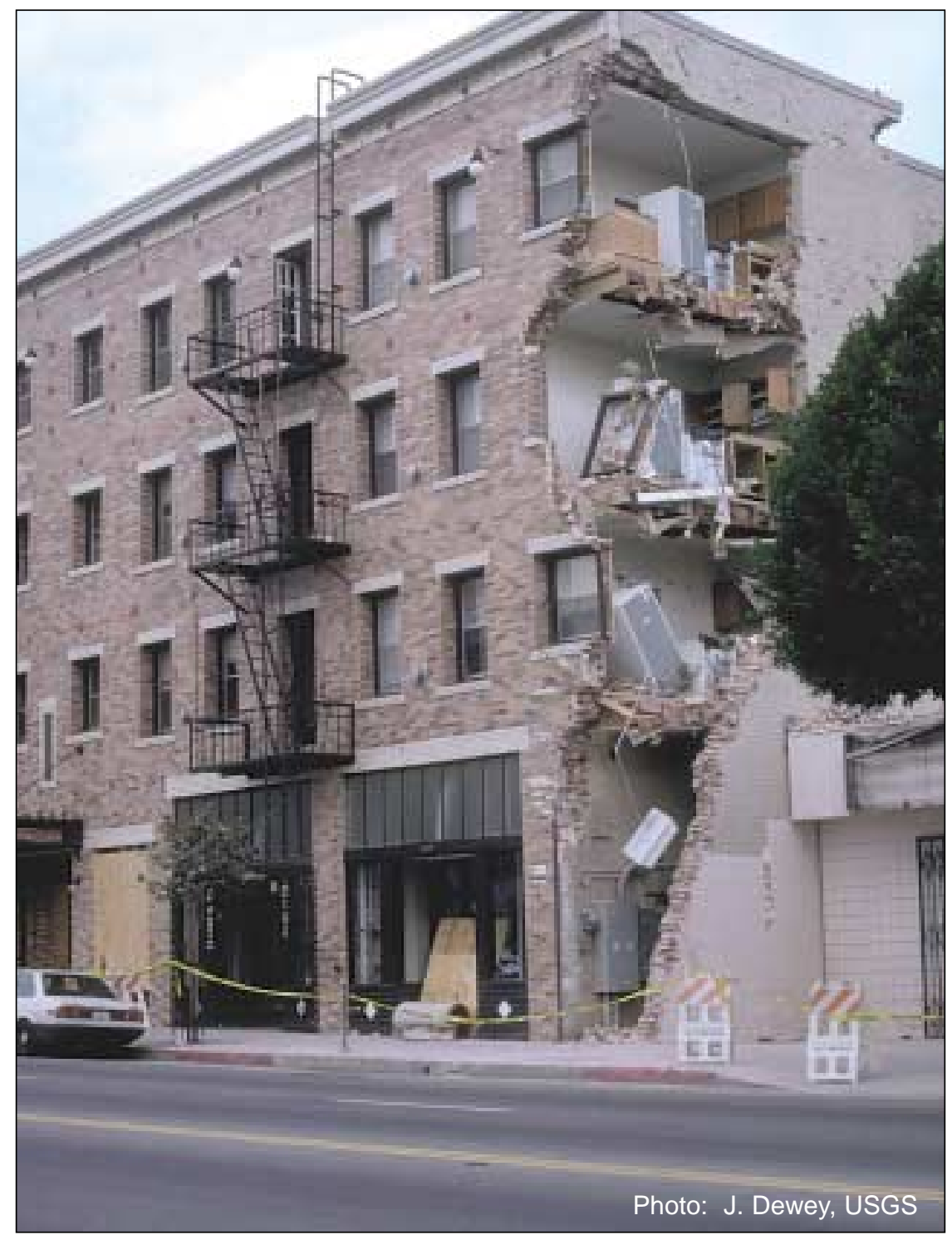

This report is preliminary and has not been reviewed for conformity with U.S. Geological Survey editorial standards nor with the North American Stratigraphic Code. Any use of trade names in this publication is for descriptive purposes only and does not imply endorsement by the U.S. Government.

\section{U.S. Department of the Interior}

U.S. Geological Survey 


\section{Introduction}

$\mathrm{E}$ arthquake-generated seismic waves propagating to the Earth's surface from their source reflect back into the Earth when that surface is free from tractions. However, if there is a built structure on the Earth's surface seismic waves will propagate into the structure causing it to vibrate.

Generally, engineers calculate the response of structures to earthquake loading by vibration methods.

We use a wave-propagation approach following the basic framework of Safak's (1999) finite-difference discrete time domain wave-propagation model of seismic response of multistory buildings and present a dynamic finite-element analysis of wave propagation in a multistory building founded on a layered substrate. Like Safak (1999), we assume that the building and the underlying soil and rock layers can be modeled as coupled continua with each story acting as another layer in response to vertically propagating waves emanating from a source within the underlying soil layers. Plane conditions are assumed in our finite-element model as in Safak's (1999) model. However, we include such 2-dimensional effects as rocking that were omitted in Safak's (1999) model.

We begin with brief descriptions of the dynamic finite-element program used for the calculations, SAP 2000@, the geometry of the finite-element mesh used, and the elastic properties assumed for the multistory building and the underlying layered substrate. Next, results from our finite-element calculations on the response of a 10-story building and underlying substrate to a vertically incident plane wave are described and compared with the discrete time domain results of Safak (1999) for a similar problem. The work in this report is preliminary and is intended to be an investigation of the potential of finite-element wave propagation methods for modeling seismic response of multistory buildings.

\section{SAP 2000@ and the configuration for modeling building/soil response}

S AP 2000@ is a well-known finite element program capable of static and dynamic 2- and 3-dimensional analysis of solids and structures. For modeling wave propagation in the multistory building and layered substrate we use planestrain nine-node quadrilateral solid elements and SAP 2000@'s algorithm for solution of the dynamic equilibrium equations by superposition of loaddependent Ritz-vector modes (Wilson and others 1982, Bayo and Wilson, 1984).

Spatial discretization of a linearly elastic body into finite elements with nodes at corners and, possibly, other locations on the elements, and application of LaGrange's equations of motion leads to the global dynamic equilibrium equations,

$$
\mathrm{Mu}(t)+\mathrm{C} \dot{\mathrm{u}}(t)+\mathrm{Ku}(t)=\mathrm{F}(t)
$$

These equations, in matrix form, apply at a time $t$, and have, respectively, $\mathbf{M}, \mathbf{C}$, and $\mathbf{K}$ as the mass, damping and stiffness matrices. Time-dependent nodal accelerations, velocities, and displacements are, respectively, $\ddot{\mathbf{u}}, \dot{\mathbf{u}}$, and $\mathbf{u}$. The $\mathbf{M}, \mathbf{C}$, and $\mathbf{K}$ matrices are square $n$ by $n$ matrices where $n$ is the number of possible nodal point displacements. Nodal accelerations, velocities, and displacements are 1 by $n$ matrices and $\mathbf{F}(t)$ is a 1 by $n$ matrix of timedependent applied loads.

SAP 2000@'s algorithm for solution of the dynamic equilibrium equations by superposition of loaddependent Ritz-vector modes is based on separation of variables. It is assumed that the solution is of the form,

$\mathbf{u}(t)=\Phi Y(t)$

with,

$\dot{\mathbf{u}}(t)=\Phi \dot{\mathbf{Y}}(t)$

and

$\ddot{\mathrm{u}}(t)=\Phi \ddot{\mathrm{Y}}(t)$

where $\Phi$ is an $n$ by $m$ space- and load-dependent matrix of Ritz vectors and $\mathbf{Y}(t)$ is a 1 by $m$ timedependent matrix.

Solution of equation 1 requires the Ritz vectors to satisfy the orthogonality conditions,

$\Phi^{\mathrm{T}} \mathrm{M} \Phi=\mathrm{I}$

and

$\Phi^{\mathrm{T}} \mathrm{K} \Phi=\Omega^{2}$ 
where $\Phi^{\mathrm{T}}$, is the transpose of $\Phi, \mathrm{I}$ is the diagonal identity matrix, and $\Omega^{2}$ is a diagonal matrix of squared vibration frequencies, $\omega_{m}^{2}$. Since the calculation of Ritz vectors that satisfy equations 5 and 6 is somewhat involved, an outline of the method used in SAP 2000@ is presented in the Appendix to this report.

Substitution of 2, 3 and 4 into equation 1 and premultiplying the resulting equation by the transpose of the Ritz vector, $\Phi^{\mathrm{T}}$, and using equations 5 and 6 leads to the set of $m$ time-dependent uncoupled ordinary differential equations given by

$\mathrm{I} \ddot{\mathrm{Y}}(t)+\Lambda \dot{\mathrm{Y}}(t)+\Omega^{2} \mathbf{Y}(t)=\mathbf{R}(t)$

where $\mathbf{R}(t)=\Phi^{\mathrm{T}} \mathbf{F}(t)$ and $\Lambda$ is the damping matrix. The damping matrix is assumed to be diagonal with terms of the form $2 \zeta_{m} \omega_{m}$ where $\zeta_{m}$ is the damping ratio that is expressed in terms of percent of critical damping.

Alternatively, equation 7 can be written in subscript notation as

$\ddot{y}(t)_{m}+2 \zeta_{m} \omega_{m} \dot{y}(t)_{m}+\omega_{m}^{2} y(t)_{m}=R_{m}(t)$

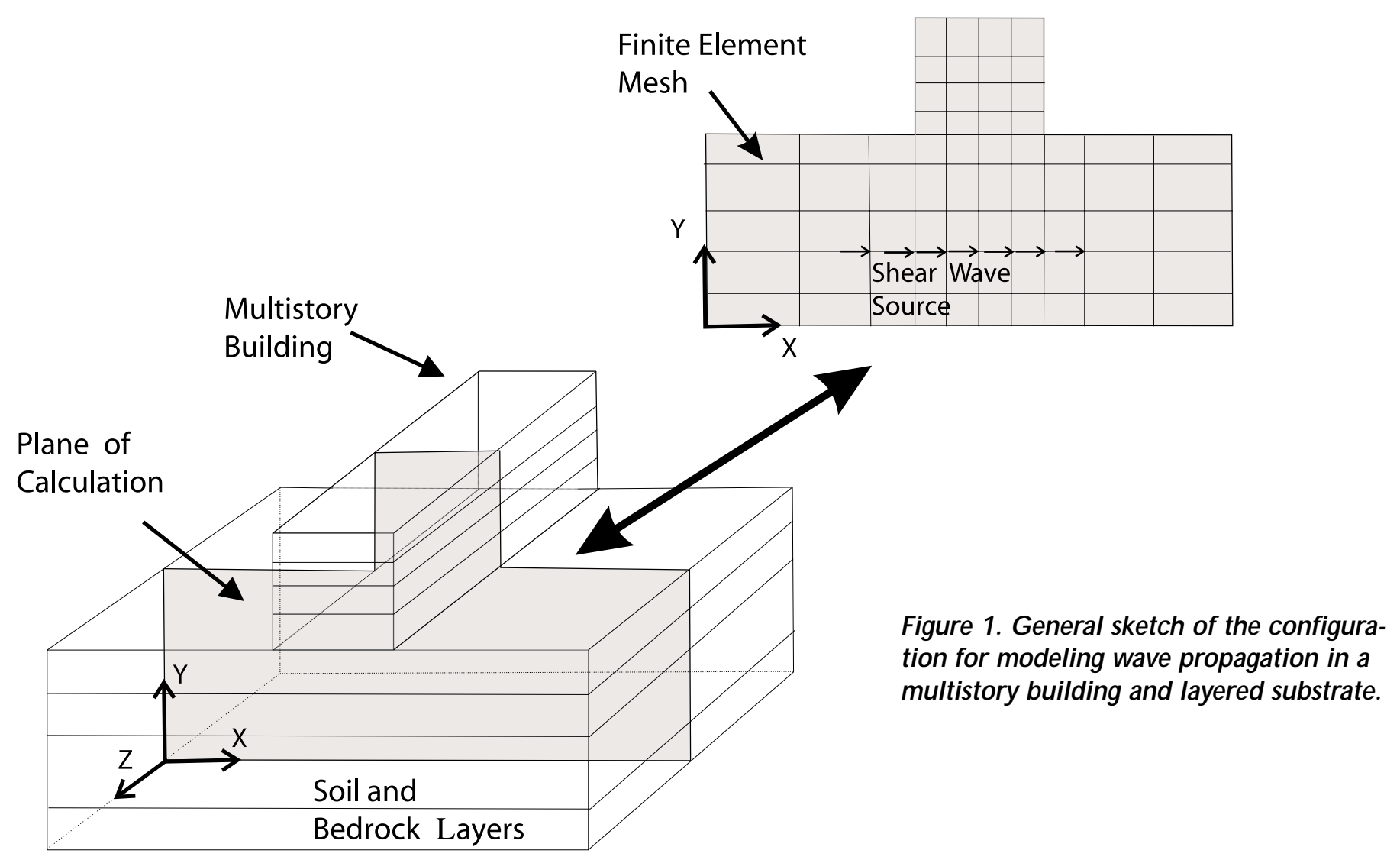

Equation 8 is a modal equation for mode $m$ and $\omega_{m}$, and $\zeta_{m}$ are the frequencies and the damping ratio for that mode. For a small time increment, the modal loading $\boldsymbol{R}_{\boldsymbol{m}}(\boldsymbol{t})$ is taken to be approximately linear and equation 8 is solved exactly for modal displacement, velocity, and acceleration in each time step. Finally, SAP 2000@ uses equations 2 through 4 and the modal displacements, velocities, and accelerations calculated at each time step to give displacements, velocities, and accelerations at unconstrained nodes throughout the finite-element model.

The mode superposition method provides a robust method for solving elasto-dynamic problems. This is because the method, based on the construction of a set of orthogonal vectors, reduces the large set of global equilibrium equations (equation 1 ) to a much smaller set of uncoupled second order linear differential equations which can be rapidly and exactly solved.

Figure 1 is a general sketch of the configuration for our finite-element model of wave propagation in a multistory building and layered substrate. We assume plane strain conditions, that is, all planes parallel to the plane of calculation in figure 1 
deform identically. Mathematically this means that there can be nodal displacements in the $\mathrm{x}$ - and $\mathrm{y}$ directions, that is, $u_{x}$ and $u_{y}$ can be non-zero, while nodal displacements in the $z$-direction, $u_{z}$, are zero.

Vertically propagating waves emanate from the shear wave source shown in figure 1 . The source is situated so that the generated wave front will become plane and vertically incident at the interface between the multistoried building and its substrate.

The finite-element mesh for the plane-strain model of the building and layered soil is shown in figure 2 . Excluding the 10-story building, the finite element mesh extends from 0 to 4,000 meters in the $x$-direction and from 0 to 1,950 meters in the Y-direction and is laterally restrained. The 10-story building is 20 meters wide and 36 meters high. Elastic material properties for the soil layers and building stories, identified by number, are given in table I. Each

\section{Figure 2. The finite-element mesh for the plane-strain model of the building and layered soil. Elastic material properties for the soil layers and building stories identified by number in this figure are given in table $I$. The locations of the source of the shear wave and nodes $A$, $B$, and $C$ (which are discussed in the text) are also shown.}

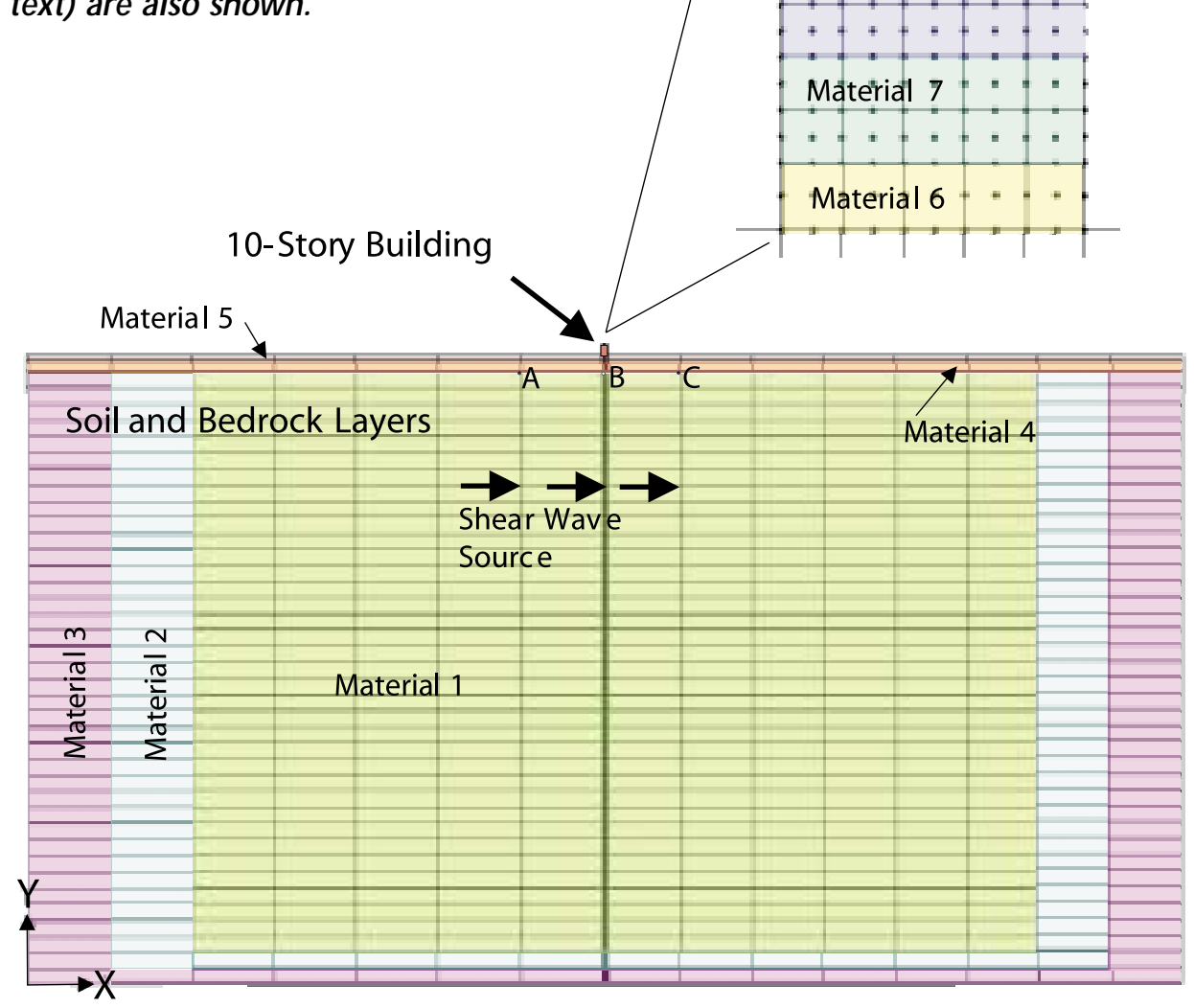

quadrilateral in figure 2 represents a nine-node plane finite element. For simplicity, element nodes are shown only in the detailed view of the 10-story building. Finally, the locations of the source of the vertically propagating waves and nodes $(A, B$, and C) that are used to check whether the generated wave approaches the building foundation as a vertically-incident plane wave are indicated in figure 2 .

Elastic material properties for the soil layers and building stories identified by number in figure 2 and given in table I are similar to those given by Safak (1999). Three different materials are given for the substrate. Material 1 has similar elastic properties to the bedrock in Safak's (1999) model. Materials 2 and 3 have progressively higher elastic moduli that, along with the application of 10 percent damping to all Ritz-vector modes, yields, as will be seen subsequently in the calculated response, reduced displacements and minimal reflection of waves from the restrained model boundaries.

Safak (1999) gives masses, shear stiffnesses, and shear-wave velocities for the building properties. The finite-element model requires density, Poisson's ratio, and Young's modulus. We calculate densities for the stories from the masses and the dimensions of each story given by Safak (1999). Consistent with our plane strain assumption, unit thickness is taken for the third dimension. Poisson's ratio is assumed to be one-third for all layers and Young's modulus is obtained from the calculated densities and Safak's (1999) shear wave velocities using $E=2 \rho[1+\sigma] \mathbf{V}_{\mathbf{s}}{ }^{2}$, where $E$ is Young's modulus, $\rho$ is density, and $v_{S}$ is shear wave velocity. The thickness of soil layers 1 and 2 and dimensions of the building stories are the same as those given by Safak (1999). Finally, 100 Ritz-vector modes are used in our calculation. 
Table I. Elastic material properties for the soil layers and building stories

\begin{tabular}{ccccc}
\hline $\begin{array}{c}\text { Material } \\
\text { Number }\end{array}$ & $\begin{array}{c}\text { Young's } \\
\text { Modulus Pa }\end{array}$ & $\begin{array}{c}\text { Poisson's } \\
\text { Ratio }\end{array}$ & $\begin{array}{c}\text { Density } \\
\mathbf{K g} / \mathbf{M}^{\mathbf{3}}\end{array}$ & $\begin{array}{c}\text { Shear Wave } \\
\text { Velocity Km/Sec }\end{array}$ \\
\hline 1-Bedrock & $2.99 \mathrm{E}+10$ & 0.333 & 2800 & 2000 \\
Material-2 & $1.00 \mathrm{E}+11$ & 0.333 & 2800 & 3660 \\
Material-3 & $1.00 \mathrm{E}+12$ & 0.333 & 2800 & 11574 \\
$4-2^{\text {nd }}$ Soil Layer & $1.67 \mathrm{E}+09$ & 0.333 & 2500 & 500. \\
$5-1^{\text {st }}$ Soil Layer & $2.35 \mathrm{E}+08$ & 0.333 & 2200 & 200 \\
$6-\mathbf{1}^{\text {st }}$ Story & $2.40 \mathrm{E}+07$ & 0.333 & 177 & 225 \\
$7-2^{\text {nd }} \& 3^{\text {rd }}$ Story & $3.50 \mathrm{E}+07$ & 0.333 & 250 & 229 \\
$8-4^{\text {th }} \& 5^{\text {th }}$ Story & $3.00 \mathrm{E}+07$ & 0.333 & 250 & 213 \\
$9-6^{\text {th }} \& 7^{\text {th }}$ Story & $2.60 \mathrm{E}+07$ & 0.333 & 250 & 196 \\
$10-8^{\text {th }}$ 9 $9^{\text {th }}$ Story & $2.10 \mathrm{E}+07$ & 0.333 & 250 & 177 \\
$11-10^{\text {th }}$ Story & $1.25 \mathrm{E}+07$ & 0.333 & 143 & 181
\end{tabular}

\section{Calculated response of the 10-story building and underlying soil}
Safak (1999, fig. 5) presents accel- eration time histories for the first soil and the 10 stories and founda- tion of the building. Our figures 5a-5e show finite-element-calcu- lated acceleration time histories for the 1st soil, the foundation, 1st floor, 5th floor, and 10th floor. With the exception of the founda- tion, all time histories in figure 5 are from nodes in the center of the 1 st soil layer and the center of each story. The foundation time history is from the center node at the interface between the 1st soil and 1 st story (see fig. 2). Safak's acceleration time histories are quite similar to those shown in our figure 5 . In both cases, acceleration amplitudes increase upwards. Figure 6 shows finite-element-calculated Fourier amplitudes of accelerations for the 1st soil, the foundation, 1st floor, 5th floor, and 10th floor. Again, acceleration amplitudes generally increase with height.

Figure 7 shows the finite-element-calculated x-displacement histories for central nodes of the foundation, fifth floor, and roof of the 10-story building. Note the increase of displacement amplitude with height in the building.

Figure 8 shows the finite-element-calculated $\mathrm{y}$-displacement of nodes at the left corner, center, and right corner of the building roof. Rocking which was omitted in Safak's (1999) model is evident. However, the $y$-displacements in figure 8 are an order of magnitude smaller than the $\mathrm{x}$-displacements shown in figure 7.

\section{Concluding discussion}

Like the finite-difference wave-propagation method introduced by Safak (1999), dynamic finite-elementwave propagation methods have considerable potential for modeling seismic response of multistory buildings. Although the model presented here is 2-dimensional, 3-dimensional finite-element models are easily implemented using existing software such as SAP 2000@. Similarly, nonlinear options in finiteelement software such as SAP 2000(C can be included in models of seismic building response.
Figure 3. Applied horizontal nodal displacement history in the source area. 

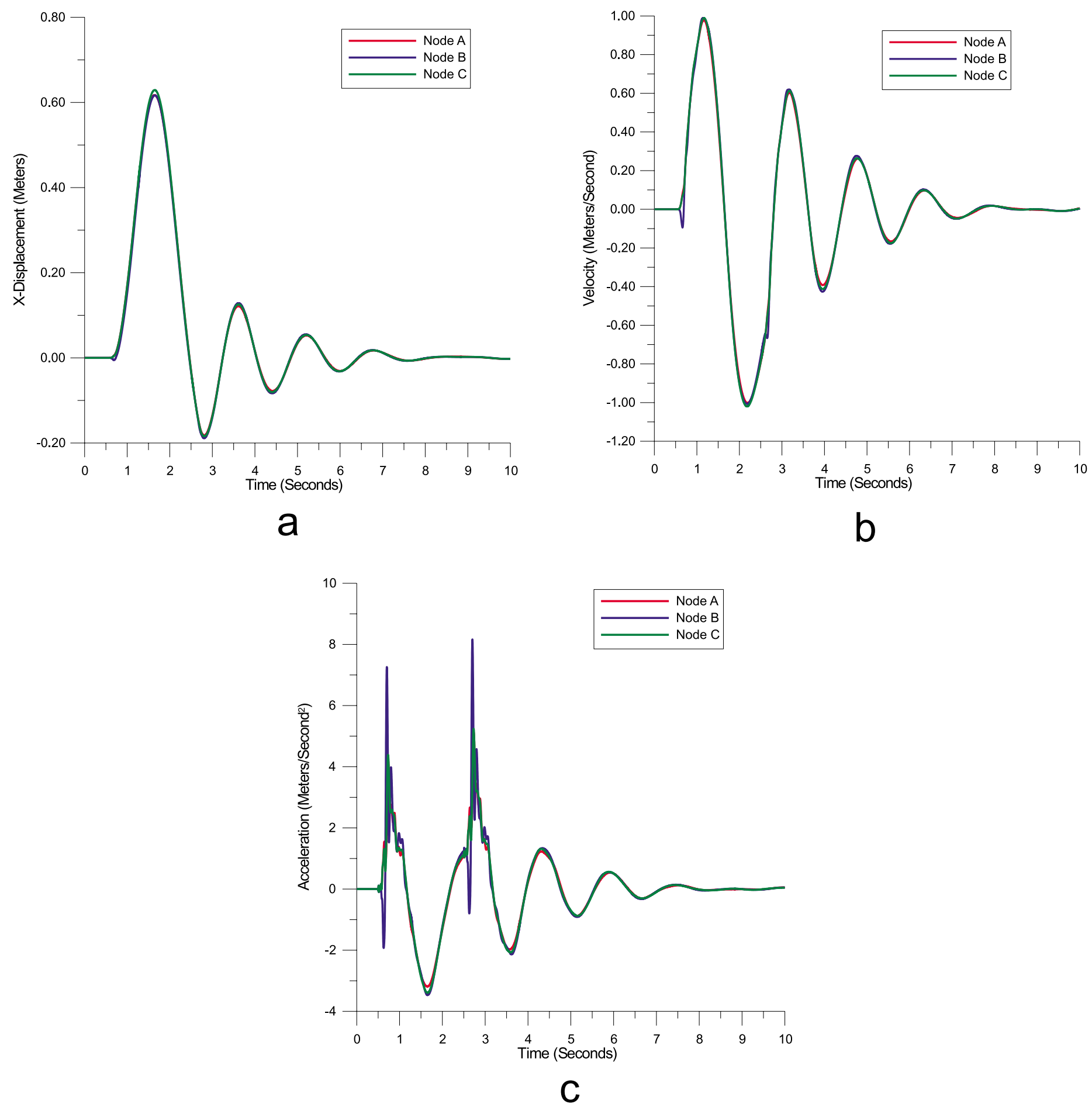

Figure 4. Horizontal displacement (4a), velocities (4b), and accelerations (4c) at nodes A, B, and C in the bedrock-soil interface. 

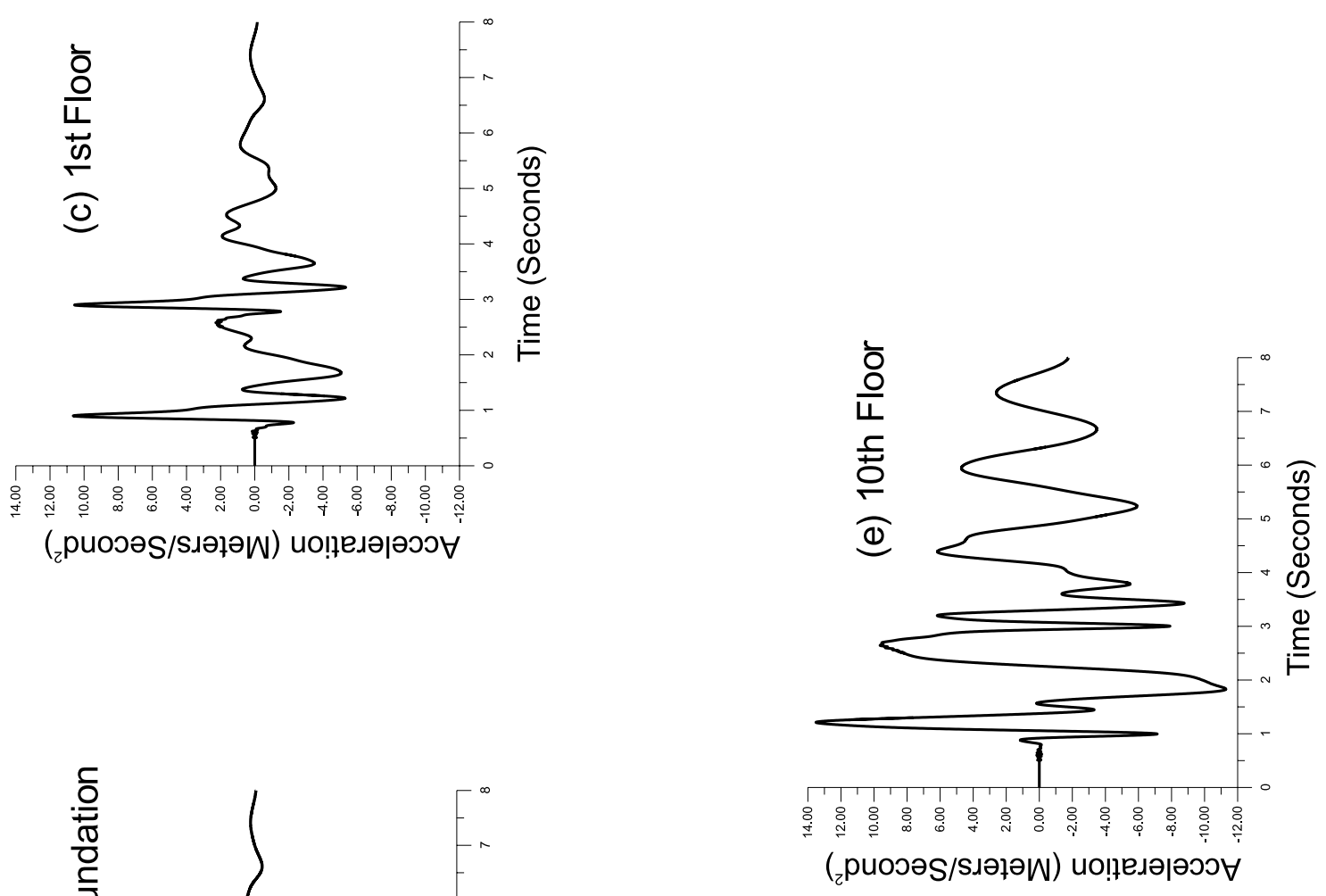

8
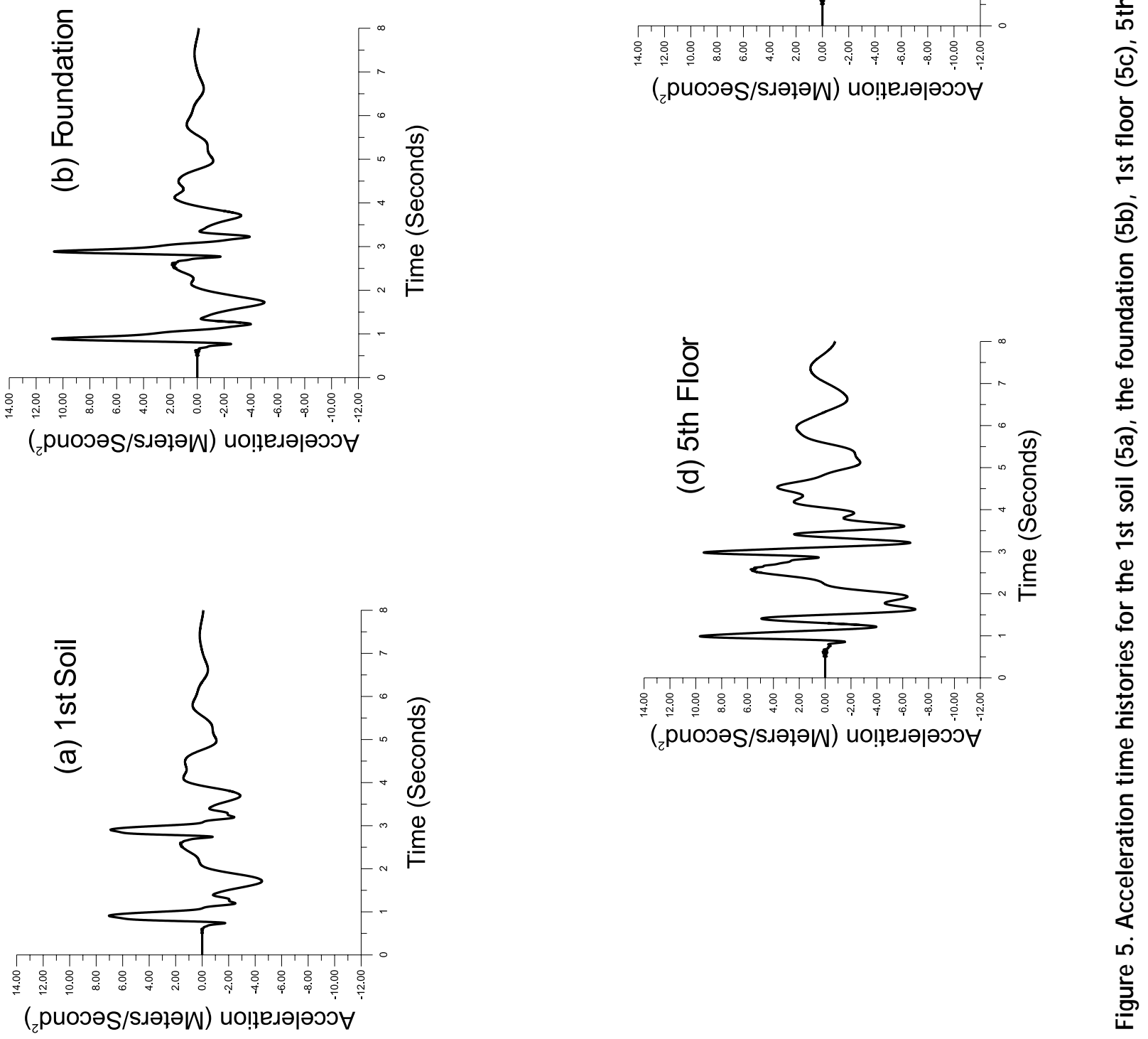


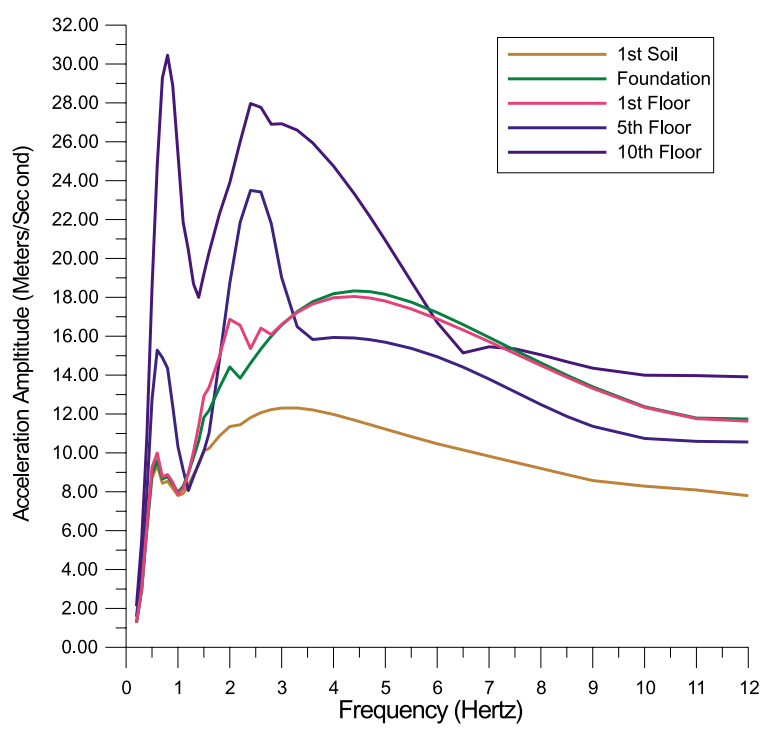

Figure 6. Fourier amplitudes of accelerations for the 1st soil, the foundation, 1st floor, 5th floor, and 10th floor. 10 percent damping for all modes.

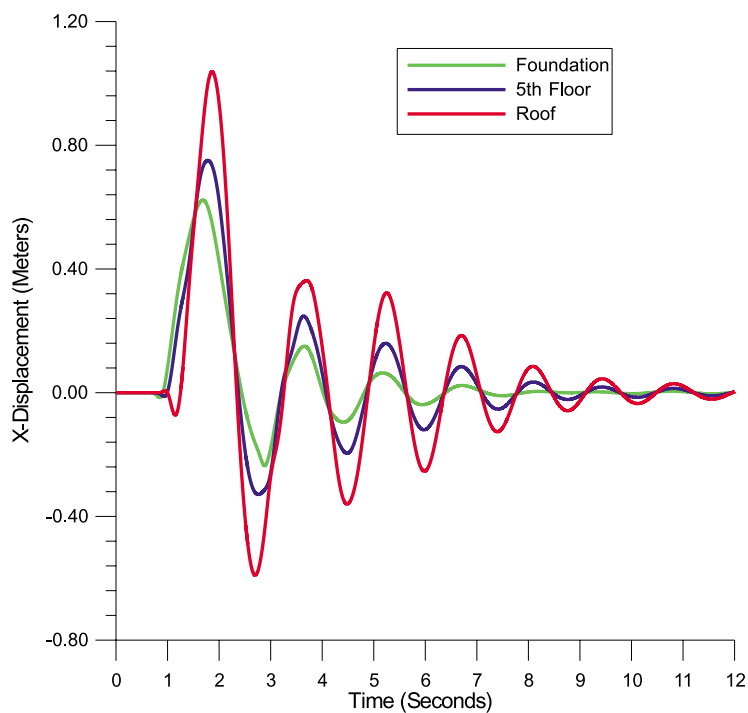

Figure 7. X-displacement histories for central nodes of the foundation, 5 th floor, and roof of the 10-story building

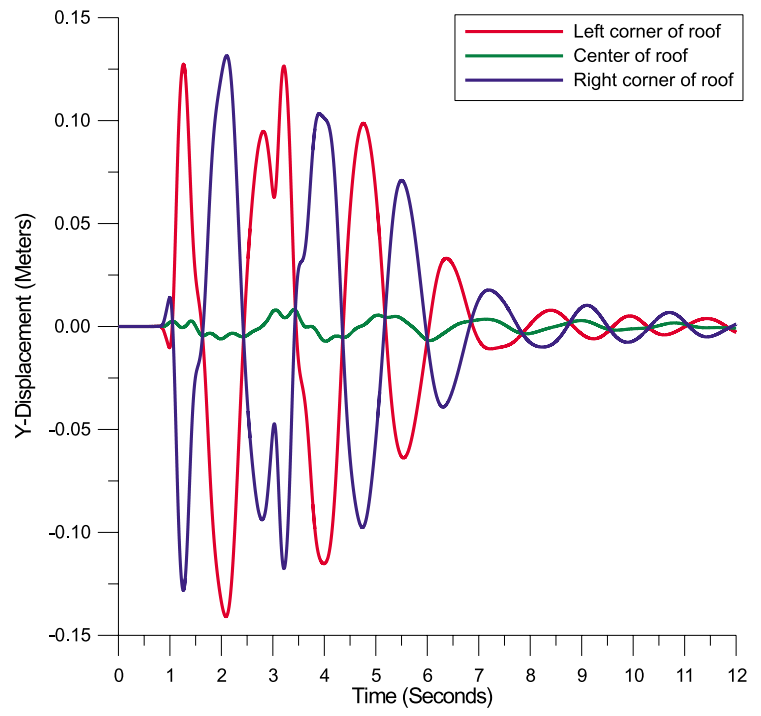

Figure 8. Y-displacement of nodes at the left corner, center, and right corner of the building roof. 


\section{References}

Bayo, E. and Wilson, E.L., 1984, Use of Ritz vectors in wave propagation and foundation response: Earthquake Engineering and Structural Dynamics, v. 12, p. 499-505.

Safak, E., 1999, Wave-propagation formulation of seismic response of multistory buildings: ASCE Journal of Structural Engineering, v. 125, no. 4, p. 426-437.
Wilson, E.L., Yuan, M-W, and Dickens, J.M., 1982, Dynamic analysis by direct superposition of Ritz vectors: Earthquake Engineering and Structural Dynamics, v. 10, p. 813-821.

\section{Appendix: Calculation of Ritz vectors in SAP 2000@}

Calculation of Ritz vectors in SAP 2000@ is based on the idea that the dynamic response of an elastic medium depends on the spatial distribution of loads. In the absence of damping the dynamic equilibrium equations (equations 1 ) become

$\mathbf{M u}(t)+\mathbf{K u}(t)=\mathbf{P}(t)$

Here $\mathbf{P}(\mathbf{t})=\mathbf{f g}(\mathbf{t})$ with $\mathbf{f}$ a vector of time-independent loads and $\mathbf{g}(\mathrm{t})$ a function of time only that can be expanded into a Fourier series of sine and cosine functions. Equation A1 for a typical, but unknown, loading frequency, $\omega_{p}$, then becomes

$\mathbf{K u}=\mathbf{f}+\omega_{p}{ }^{2} \mathbf{M u}$

Because the loading frequency is unknown, equation A2 cannot be directly solved for the displacements $\mathbf{u}$, however, it can be solved by a recurrence algorithm. The procedure starts by neglecting the mass in equation $\mathrm{A} 2$, giving

$\mathrm{Ku}_{S}=\mathbf{f}$

Equation A3 is solved for $\mathbf{u}_{\boldsymbol{s}}$, the static response of the elastic medium. Then a new force vector

$\mathbf{f}_{1} \approx \mathbf{M u}_{0}$

is constructed that approximates the error caused by neglecting mass in the solution of equation A2.

Equation A4 is used to calculate a new displacement vector, $\mathbf{u}_{\mathbf{1}}$ from

$\mathbf{K} \mathbf{u}_{1}=\mathbf{f}_{1}$ and the process is repeated leading to the recurrence relation

$$
\mathbf{K u}_{1}=\mathbf{M u}_{i-1}
$$

The static response vector $\mathbf{u}_{\mathbf{s}}$ is made orthogonal to the mass and stiffness matrices resulting in a new vector $\mathbf{V}_{1}$, then the displacements in equation A5 are also made stiffness and mass orthogonal, and the process is continued until the last vector $\mathbf{V}_{i}$ is found to be orthogonal to all previously calculated vectors. This vector is normalized by $\mathbf{V}_{i}{ }^{\mathrm{T}} \mathbf{M} \mathbf{V}_{i}=\mathbf{I}$ producing a mass orthogonal Ritz vector.

Finally, solution of the eigenvalue problem

$\left\lfloor\overline{\mathbf{K}}-\Omega^{2} \mathrm{I}\right\rfloor \mathrm{Z}=0$

where $\overline{\mathbf{K}}=\mathbf{V}^{\mathrm{T}} \mathbf{K V}$ and forming the product

$\Phi=\mathbf{V Z}$

yields load dependent Ritz vectors that have the desired properties of mass and stiffness orthogonality (equations 5 and 6).

The foregoing briefly outlines the numerical procedures used in SAP 2000@ to calculate Ritz vectors. We refer the reader to Wilson and others (1982) and Bayo and Wilson (1984) for further details. 